\title{
Composição química do óleo fixo de Croton cajucara e determinação das suas propriedades fungicidas
}

\author{
Marco André A. Souza ${ }^{1}$, Sonia R. Souza ${ }^{1}$, Valdir F. Veiga Jr², Janaína K.P.C. Cortez ${ }^{3}$ Rosélia \\ de Sousa Leal ${ }^{3}$, Tereza N. Castro Dantas ${ }^{3}$, Maria Aparecida M. Maciel ${ }^{3 *}$ \\ ${ }^{1}$ Departamento de Quimica, Instituto de Ciências Exatas, Universidade Federal Rural do Rio de Janeiro, \\ 23851-970, Seropédica, RJ, Brasil, \\ ${ }^{2}$ Departamento de Química, Universidade Federal do Amazonas, 60077-000, Manaus, AM, Brasil, \\ ${ }^{3}$ Departamento de Quimica, Universidade Federal do Rio Grande do Norte, Campus Universitário, \\ 59072-970, Natal, RN, Brasil
}

\begin{abstract}
RESUMO: Este trabalho objetivou a obtenção de óleos fixos (OF) das cascas do caule de Croton cajucara via percolação [seguido de fracionamento em coluna cromatográfica $(\mathrm{CC})$ ] e fluido supercrítico (FS, gás $\mathrm{CO}_{2}$ ), bem como a avaliação das propriedades fungicidas de OF obtido pelo procedimento CC. Os óleos OF-CC e OF-FS apresentaram rendimentos significativamente diferentes; houve maior rendimento $(3,14 \%)$ de OF obtido no processo convencional (OF-CC), versus $1,2 \%$ de OF-FS. A fração $F_{1}$ após esterificação, bem como as frações não-esterificadas $F_{2}, F_{3}$ e $\mathrm{F}_{4}$ (obtidas de OF-CC seguido de outro fracionamento em CC) foram analisadas por CG-FID e CG-EM. A identificação da composição química de OF-CC foi feita por comparação com dados da biblioteca Wiley, bem como pela comparação de índices de kovats. A presença de sesquiterpenos foi comprovada na fração $F_{1}$, onde o $\alpha$-copaeno $(20,1 \%)$ e cipereno $(21,8 \%)$ foram os componentes majoritários $\left(70 \%\right.$ de percentual sesquiterpênico total). A fração $\mathrm{F}_{2}$ mostrou sesquiterpenos minoritários mais oxigenados, incluindo o linalol. $\mathrm{Na}$ fração $\mathrm{F}_{3}$ foram observados ácidos graxos, misturas de esteróis e os diterpenos bioativos trans-crotonina, cis-cajucarina B e trans-cajucarina $\mathrm{B}$, onde $40 \%$ do total desta fração, foi composta de esteróis e diterpenos; e na fração $\mathrm{F}_{4}$ foram observados majoritariamente os isômeros cis e trans-cajucarina B. O efeito biológico de OF-CC foi avaliado no desenvolvimento in vitro dos fungos fitopatogênicos Fusarium oxysporum, Rhizoctonia solani e Sclerotium rolfsii propagados em meio de cultura Batata-dextrose-agar (BDA). A resposta fisiológica, variou de acordo com o tipo de fungo testado, tendo sido observado que o OF-CC $\left(0,2 \mathrm{mg} \cdot \mathrm{mL}^{-1}\right)$ apresentou efeito fungistático no controle micelial dos fungos testados, sendo que o gênero de fungo Fusarium oxysporum foi o que sofreu efeito inibitório mais estável.
\end{abstract}

Unitermos: Croton cajucara, cascas do caule, óleo fixo, constituintes químicos, propriedades fungicidas.

\begin{abstract}
Chemical composition of the fixed oil of Croton cajucara and its antifungical properties". This paper focused on the separation of the fixed oil (FO) from the stem bark of Croton cajucara obtained from the conventional approach extraction with organic solvents [followed by chromatography column (CC)] and supercritical fluid extraction (SFE, carried out with $\mathrm{CO}_{2}$ ) and also, on the antifungical properties of the FO obtained by CC procedure (FO-CC). The FO contents were $3.14 \%$ for FO-CC versus $1.20 \%$ in the FO-SFE process. The esterified fraction $\mathrm{F}_{1}$ and nonesterified fractions $\mathrm{F}_{2}, \mathrm{~F}_{3}$ and $\mathrm{F}_{4}$ obtained from $\mathrm{FO}-\mathrm{CC}$ after a new $\mathrm{CC}$ procedure were analyzed by HRGC-MS. The identification of the chemical composition of FO-CC was made by comparison with MS literature data, computer matching with the Wiley library and by comparison of their kovats indices with the literature. Fraction $\mathrm{F}_{1}$ showed $70 \%$ of sesquiterpene components, among them $\alpha$-copaene $(20.1 \%)$ and ciperene $(21.8 \%)$ as major compounds. Fraction $\mathrm{F}_{2}$ was rich in minor oxygenated sesquiterpenes, among them linalool. Meanwhile fraction $\mathrm{F}_{3}$ showed fat acids, steroids and the bioactive clerodane type-diterpene trans-crotonin, cis-cajucarin B (c-CJC-B) and transcajucarin $\mathrm{B}$ (t-CJC-B). Fractions $\mathrm{F}_{3}$ and $\mathrm{F}_{4}$ showed as major constituents c-CJC-B and t-CJC-B with $40 \%$ of the total contents. The biological effect of the FO-CC was evaluated in the in vitro development of the phytopatogen fungi such as Fusarium oxysporum, Rhizoctonia solani and Sclerotium rolfsii. Significant inhibitory effect of the tested fungi (at $0.2 \mathrm{mg} \cdot \mathrm{mL}^{-1}$ dosage) were proved.
\end{abstract}

Keywords: Croton cajucara, stem bark, fixed oil, chemical constituents, antifungical properties. 


\section{INTRODUÇÃO}

Croton cajucara Benth (Euphorbiaceae), vulgarmente conhecido por "sacaca", representa um recurso medicinal de grande importância no tratamento e cura de várias doenças, tais como: diabetes, diarréia, malária, febre, problemas estomacais, inflamações do fígado, rins, vesículas e no controle de índices elevados de colesterol (Maciel et al., 2002a,b). O efeito gastroprotetor desta espécie foi correlacionado com os diterpenos do tipo clerodano trans-desidrocrotonina (DCTN) e trans-crotonina (CTN) (Figura 1), bem como com o óleo essencial obtido das cascas do caule deste Croton (Hiruma-Lima et al., 1999a,b, 2000a,b). Adicionalmente, estudos já comprovaram que o óleo essencial apresentou efeitos antinociceptivo e antiinflamatório (Bighetti et al., 1999). Outros tipos de atividades biológicas foram atribuídos aos clerodanos DCTN (Agner et al., 2001; Silva et al., 2001a,b; 2005) e CTN (Maciel et al., 2000;<smiles>CC1=CC(=O)C[C@H]2[C@@H]1CC[C@@H](C)[C@]21C[C@H](c2ccoc2)OC1=O</smiles>

DCTN<smiles>COC(=O)[C@@H]1[C@H]2CC(=O)C=C(C)[C@@H]2CC[C@@H]1CCc1ccoc1</smiles>

Almeida et al., 2002, 2003).

Em decorrência do Croton cajucara possuir uma grande representatividade na medicina tradicional da região amazônica do Brasil (Maciel et al., 2002a,b) e recentemente seu uso ter se expandido para outras regiões do país (Veiga Jr. et al., 2005), estamos ampliando neste trabalho, o estudo quimio-farmacológico desta espécie. Para tanto, o óleo fixo obtido das cascas do caule foi submetido a análises química e biológica. A caracterização química foi realizada por análises de cromatografia em fase gasosa com o uso de detectores de espectrometria de massas (CG-EM) e de ionização de chama (CGFID), tendo sido utilizados padrões previamente isolados do Croton cajucara. O efeito dos princípios bioativos deste óleo foi avaliado no desenvolvimento in vitro dos seguintes fungos fitopatogênicos Fusarium oxysporum, Rhizoctonia solani e Sclerotium rolfsii.
c-CJC-B



Figura 1. Clerodanos isolados previamente das cascas do caule de Croton cajucara (Maciel et al., 1998a,b, 2003).

t-CJC-B<smiles>C[C@@H]1CC(=O)C[C@H]2[C@@H]1CC[C@@H](C)[C@]21CO[C@H](c2ccoc2)C1</smiles>

CTN<smiles>COC(=O)[C@H]1CCC[C@H]2C(C)=CC(=O)C[C@H]1[C@H]2CCc1ccoc1</smiles> 


\section{MATERIAL E MÉTODOS}

\section{Material vegetal}

O material vegetal utilizado foi obtido no mercado Ver-o-peso de Belém/PA. Uma prévia identificação botânica da planta foi feita por Nelson A. Rosa do Museu Paraense Emílio Goeldi, tendo sido depositada uma exsicata no herbário deste Museu, sob código 247. A autenticidade do material vegetal foi confirmada com análises comparativas do extrato metanólico, via cromatografia de camada fina com padrões autênticos previamente isolados (Maciel et al., 1998a,b; 2003).

\section{Extração do óleo fixo}

O material vegetal adquirido comercialmente em épocas diferentes, foi seco em estufa a temperatura de $40{ }^{\circ} \mathrm{C}$, e moído em moinho de facas do tipo Willey. O pó foi submetido à extração via percolação ou por extração via fluido supercrítico (com altas pressões e temperatura moderada).

\section{Extração via fluido supercrítico}

As cascas trituradas $(109 \mathrm{~g})$ foram peneiradas (peneira com malha de 35 Mesh) obtendo-se um material com granulometria variando entre 28 a 45 Mesh (granulometria necessária para o uso no processo de extraçãodefluidosupercrítico). Opóobtido foiempacotado (em um tubo) e submetido por aproximadamente 4 horas, a um fluxo de gás $\mathrm{CO}_{2}$, com pressão variando entre 66-70 bar e temperatura de $55^{\circ} \mathrm{C}$.

\section{Extração via percolação seguido de procedimento cromatográfico}

A extração via percolação foi efetuada com metanol, tendo sido utilizados $423 \mathrm{~g}$ de material vegetal. O extrato metanólico (EM) obtido após redução do solvente, foi submetido a fracionamento cromatográfico convencional (Maciel et al., 2002a), utilizando-se como adsorvente sílica gel (70-230 Mesh) da Merck, tendo sido obtido 6 frações $\left[\mathrm{F}_{\mathrm{A}, \mathrm{B}, \mathrm{C}, \mathrm{DEF}}\right.$ eluídas com hexano/AcOEt (100:0 - 85:15\%)], como mostrado no Esquema 1. As frações $\mathrm{F}_{\mathrm{A}}$ e $\mathrm{F}_{\mathrm{B}}$ [eluídas com hexano/AcOEt (100:0 e 98:2, respectivamente)] foram reunidas e forneceram 13 $\mathrm{g}(3,14 \%)$ de óleo fixo $\left(\mathrm{OF}-\mathrm{CC}-\mathrm{F}_{\mathrm{AB}}\right)$. A reunião de frações $\mathrm{OF}-\mathrm{CC}-\mathrm{F}_{\mathrm{AB}}$ sofreu um novo procedimento cromatográfico gerando quatro novas frações (OF-CC- $\left.\mathrm{F}_{1,2,3,4}\right)$. A fração $\mathrm{F}_{1}$ foi eluída em hexano/AcOEt (100:0 - 98:2), $\mathrm{F}_{2}$ em hexano/AcOEt (95:5), $\mathrm{F}_{3}$ em hexano/AcOEt (90:10) e $\mathrm{F}_{4}$ em hexano/AcOEt $(85: 15)$. Apenas a fração $\mathrm{F}_{1}$ sofreu esterificação com diazometano.

\section{Análise da composição química}

A fração $F_{1}$ (esterificada) e as frações $F_{2-4}$ (nãoesterificadas) foram analisadas por CG-FID e CG-EM, utilizando um cromatográfo de fase gasosa HP modelo 5890 equipado com um detector de ionização de chama a $300{ }^{\circ} \mathrm{C}$ e injetor em modo split $(1: 20)$ na temperatura de $270{ }^{\circ} \mathrm{C}$. As análises por CG-EM foram realizadas em um cromatográfo HP modelo 5880 acoplado a um espectrômetro de massas HP modelo 5897A, operado no modo de impacto de elétrons $(70 \mathrm{eV})$ com analisador quadrupolo. No espectrômetro de massas a escala de varredura foi de 40 a 600 a.m.u.. Foi utilizado hidrogênio altamente puro como gás carreador em uma velocidade linear de $2 \mathrm{~mL} \mathrm{~min}{ }^{-1}$. A temperatura do forno foi programada de $110{ }^{\circ} \mathrm{C}$ a $160{ }^{\circ} \mathrm{C}$ em $2{ }^{\circ} \mathrm{C} \min ^{-1} \mathrm{e}$, em seguida, de $290{ }^{\circ} \mathrm{C}$ a $5{ }^{\circ} \mathrm{C} \mathrm{min}^{-1}$, seguida de isoterma de 5 minutos. Em todas as análises foi utilizada coluna capilar de sílica de $25 \mathrm{~m}$ de comprimento, $0,25 \mathrm{~mm}$ de i.d. e película de $0,25 \mathrm{~mm}$ de SE-54 da fase estacionária (5\% de fenil e $1 \%$ de vinil em metil silicone). As identificações foram realizadas por comparação dos dados de cromatografia (índices de retenção) e espectrometria de massas obtidos de padrões, da literatura e de espectrotecas (Wiley 275).

\section{Desenvolvimento in vitro de fungos fitopatogênicos}

Os fungos Fusarium oxysporum, Rhizoctonia solani e Sclerotium rolfsii foram obtidos junto a micoteca do setor de fitopatologia da UFRRJ e propagados em meio de cultura Batata-dextrose-agar (BDA). Para a solubilização do óleo fixo testado (OF-CC proveniente da reunião de frações $\mathrm{F}_{\mathrm{AB}}$ ) utilizou-se dimetilsulfóxido (DMSO), tendo sido efetuado um ensaio prévio para determinar a concentração de DMSO menos tóxica aos fungos. Para o preparo da solução teste, diluiram-se 20 mg.mL ${ }^{-1}$ do OF-CC em DMSO. Em seguida, três frascos contendo o meio de cultura BDA foram fundidos e os tratamentos preparados continham: a) controle positivo (constituído do meio de cultura BDA), b) testemunha padrão [meio de cultura mais $1 \%\left(\mathrm{v} \cdot \mathrm{v}^{-1}\right)$ de $\mathrm{DMSO}$ ] e c) tratamento teste [meio de cultura mais $1 \%\left(\mathrm{v} \cdot \mathrm{v}^{-1}\right)$ da solução teste (previamente preparada), de modo que a concentração do óleo fixo no meio de cultura foi de 0,2 $\left.\mathrm{mg} \cdot \mathrm{mL}^{-1}\right]$. Os meios de cultura utilizados foram preparados com antibiótico de largo espectro (Gentamicina $\left.{ }^{\circledR}\right)$, tendo sido vertidos nas placas de Petri $(4 \mathrm{~mm} \pm 1 \mathrm{~mm}$ de diâmetro) e logo após a solidificação, foram repicados fragmentos de BDA no centro destas placas que continham estruturas reprodutivas dos fungos Fusarium oxysporum, Rhizoctonia solani e Sclerotium rolfsii. As placas de Petri foram lacradas com filme plástico, identificadas e armazenadas em uma câmara de crescimento, com luz do dia e temperatura de $28^{\circ} \mathrm{C} \pm 1^{\circ} \mathrm{C}$, durante cinco dias, após $\mathrm{o}$ inicio do ensaio. Os dados foram coletados pela média do diâmetro da colônia nos dois sentidos ortogonais, os resultados foram submetidos à análise de variância e as médias comparadas pelo teste de Fisher LSD com 5\% de significância (Souza et al., 2003). 


\section{RESULTADOS E DISCUSSÃO}

Os óleos fixos obtidos via cromatografia em coluna (OF-CC) e fluido supercrítico (gás $\mathrm{CO}_{2}$ ) apresentaram rendimentos significativamente diferentes. A comparação feita entre as duas extrações [via percolação (seguido de CC) e fluido supercrítico] revelou que houve um maior rendimento de óleo fixo obtido no processo convencional (OF-CC), tendo sido obtido $13 \mathrm{~g}(3,14 \%)$ de óleo fixo (partindo-se de $413 \mathrm{~g}$ de cascas), versus 1,2\% de óleo fixo extraído no processo via fluido supercrítico (1,34 g; partindo-se de $109 \mathrm{~g}$ de cascas). Estes dados poderiam estar correlacionado com questões sazonais [variações que dependem da época e horário de coleta, dentre outros fatores (Maciel et al., 1998b, 2002a)], já que a fonte vegetal utilizada em cada experimento é comercializada no mercado Ver-o-peso (Belém/PA) e foi adquirida em épocas diferentes. A extração com fluido supercrítico minimiza gastos e tempo de trabalho, desta forma, mesmo que forneça rendimentos menores, tornase uma alternativa viável na extração de óleos em geral. Como exemplo do uso da extração via fluidos supercríticos objetivando redução de custos, podemos citar processos de decafeinização de café e chá praticados na indústria Alemã, Italiana e dos EUA (Zacchi et al., 2006).

Até o presente momento, não há relatos de estudos que tenham efetuado caracterização química de óleo essencial ou fixo obtidos das cascas do caule de



Esquema 1. Procedimentos de extração e fracionamento em coluna cromatografia para obtenção do óleo fixo das cascas do caule de Croton cajucara. 
Tabela 1: Substâncias voláteis da fração $\mathrm{F}_{1}$ detectadas por CG-EM e confirmadas por seus dados de IR por CG-FID.

\begin{tabular}{lcccc}
\hline Substâncias detectadas & $\%^{*}$ & $\%^{* *}$ & IR obtido & IR lit. \\
\hline Cicloisosativeno & 2,3 & 3,3 & 1370 & - \\
$\alpha$-Copaeno & 13,9 & 20,1 & 1376 & 1369 \\
Cipereno & 15,1 & 21,8 & 1398 & 1398 \\
$\alpha$-Bergamoteno & 1,6 & 2,3 & 1408 & 1436 \\
2,6-Dimetil-6-(4-metil-3-pentenil) biciclo[3.1.1] hep-2-eno & 8,3 & 12,0 & 1415 & - \\
cis-Cariofileno & 5,6 & 8,1 & 1438 & 1426 \\
allo-Aromadendreno & 2 & 2,9 & 1455 & 1463 \\
$\alpha$-Longipineno & 3,6 & 5,2 & 1467 & 1357 \\
$\delta$-Guaieno & 1,4 & 2,0 & 1486 & 1502 \\
$\alpha$-Muuroleno & 2,2 & 3,2 & 1488 & 1503 \\
$\delta$-Cadineno & 6,7 & 9,7 & 1509 & 1524 \\
Nerolidol & 2,2 & 3,2 & 1545 & 1562 \\
Junipeno & 1,4 & 2,0 & 1572 & - \\
Cadaleno & 3 & 4,3 & 1636 & 1637 \\
\hline Total & 69,3 & 100,0 & & \\
\hline
\end{tabular}

* Porcentagem observada pela integração

** Porcentagem normalizada para a constituição dos sesquiterpenos

IR obtido = Índice de Retenção obtido nas análises realizadas

IR lit. = Índice de Retenção observado na literatura, obtido nas mesmas condições de análises

Tabela 2. Substâncias voláteis da fração $\mathrm{F}_{2}$ detectadas via CG-EM.

\begin{tabular}{ll}
\hline Substâncias detectadas & $\%$ \\
\hline Linalool & 0,6 \\
Borneol & 0,5 \\
$p$-cimeno- $\alpha$-ol & $\mathrm{t}$ \\
Propanoato de linalila & $\mathrm{t}$ \\
Elemicina & 2,3 \\
Nerolidol & 1,0 \\
Espatulenol & 23,8 \\
Viridiflorol & 8,6 \\
\hline Total & 36,8 \\
\hline
\end{tabular}

Croton cajucara, apenas cita-se que o óleo essencial das cascas não apresenta traços de DCTN e é constituído de sesquiterpenos (sem divulgação da identificação química) considerados como responsáveis pelas atividades antiulcerogênica (Hiruma-Lima, et al., 1999a, 2000a), antiinflamatória e antinociceptiva (Bighetti et al., 1999). Com relação ao óleo essencial de folhas de Croton cajucara, sabe-se que é rico em linalol (Araújo et al., 1971; Lopes et al., 2000) e possui atividades antimicrobiana (Alviano et al., 2005) e antileishmanial (Rosa et al., 2003). O óleo essencial das partes aéreas desta planta, também mostrou-se rico em linalol (Lemos et al., 1999).
Neste trabalho, a fração $\mathrm{F}_{1}$ esterificada, bem como as frações não-esterificadas $\mathrm{F}_{2}, \mathrm{~F}_{3}$ e $\mathrm{F}_{4}$ (obtidas via procedimento cromatográfico, Esquema 1) foram analisadas por CG-FID e CG-EM. A identificação dos constituintes químicos destas frações foi efetuada por comparação dos espectros de massas obtidos com os dados da espectroteca Wiley e dos índices de retenção, calculados a partir da injeção de uma série de alcanos lineares (C13 - C18), tendo sido comprovada na fração $F_{1}$ a presença de sesquiterpenos (Tabela 1), onde o $\alpha$-copaeno $(20,1 \%)$ e cipereno $(21,8 \%)$ foram os componentes majoritários. Nesta fração também foram observados ésteres metílicos e etílicos. Os sesquiterpenos 


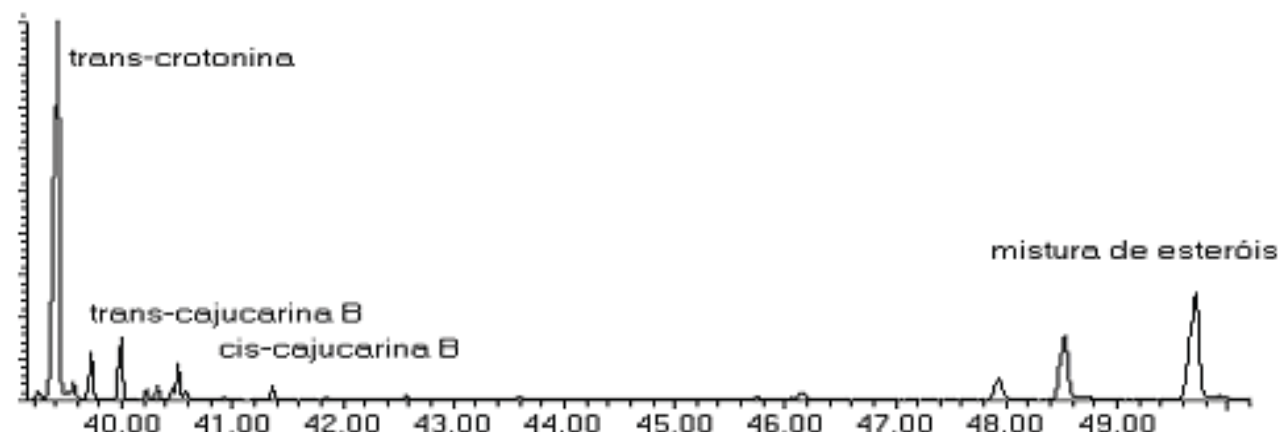

Figura 2. Expansão do cromatograma da fração $\mathrm{F}_{3}$, evidenciando clerodanos e esteróis detectados.

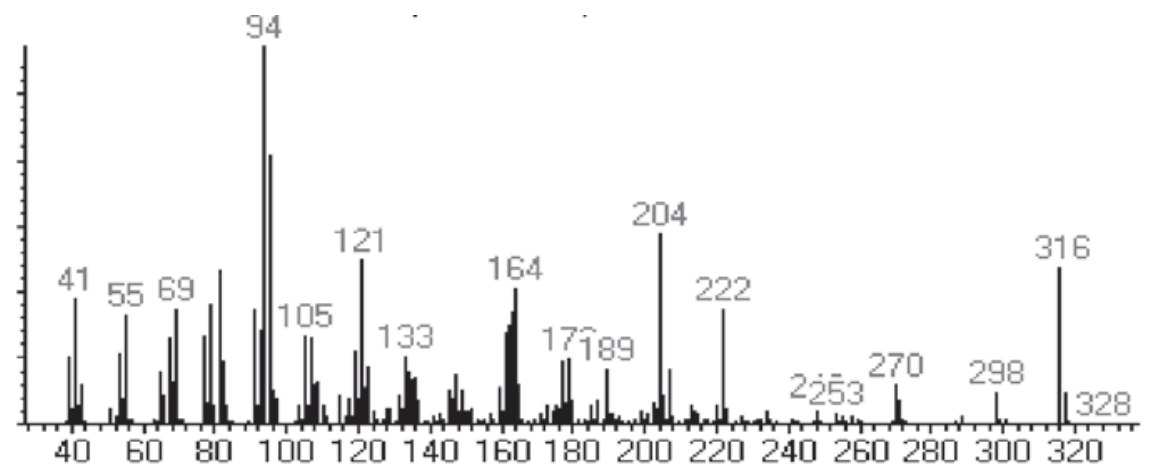

Figura 3. Espectro de massas do clerodano trans-crotonina, obtido no cromatograma da fração $\mathrm{F}_{3 .}$.<smiles>CC[CH-]CCC(C)C</smiles>

$[\mathrm{M}]^{+} 316$

$(39 \%)$<smiles>C=C1CCC2C(C)CC(=O)CC2C1=C=O</smiles>

$(51 \%)$<smiles>CC1CC(=O)CC2C(C(=O)O)=C([I+])CCC12</smiles>

$\mathrm{m} / \mathrm{z} 222$

$(22 \%)$<smiles>O=Cc1ccoc1</smiles>

$\mathrm{m} / \mathrm{z} 95$

$(76 \%)$<smiles>C=Cc1ccoc1</smiles>

$\mathrm{m} / \mathrm{z} 94$

$(100 \%)$

$\mathrm{m} / \mathrm{z} 82$

$(48 \%)$

Esquema 2. Fragmentos propostos para a CTN. 


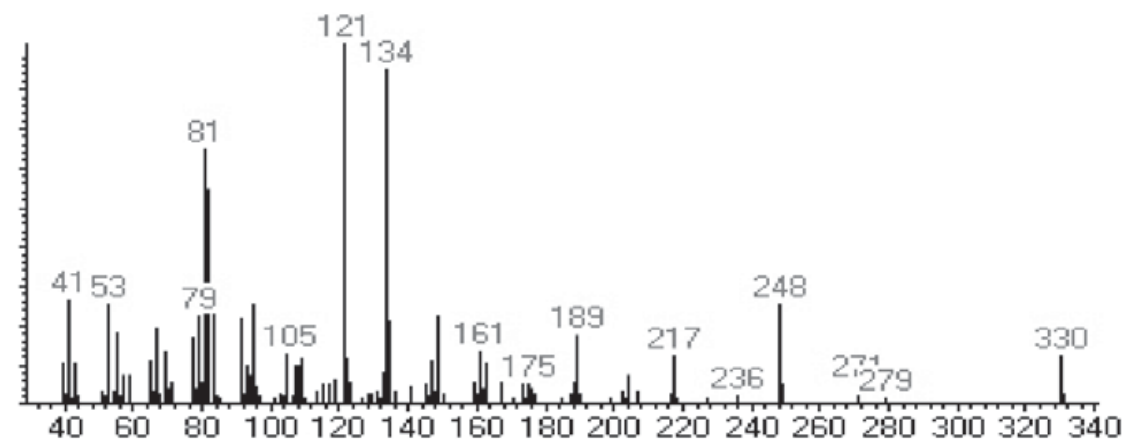

Figura 4. Espectro de massas do clerodano isomérico trans-cajucarina B, obtido no cromatograma da fração $\mathrm{F}_{3}$

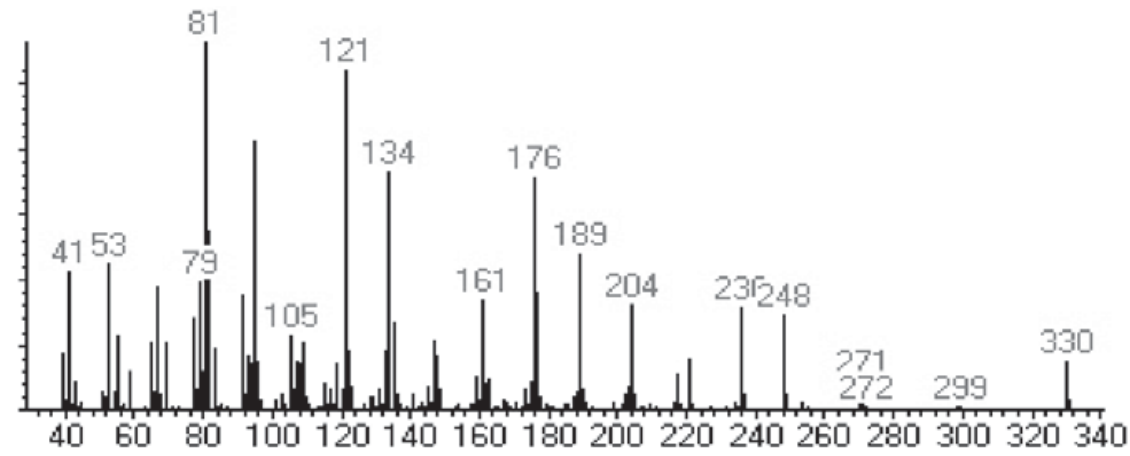

Figura 5. Espectro de massas do clerodano isomérico cis-cajucarina B, obtido no cromatograma da fração $\mathrm{F}_{3}$.

corresponderam a aproximadamente $70 \%$ da constituição química de $\mathrm{F}_{1}$. A normalização observada na Tabela 1 permite a visualização do percentual relativo entre os sesquiterpenos identificados. A fração $\mathrm{F}_{2}$ mostrou sesquiterpenos mais oxigenados (Tabela 2), onde se inclui o linalol e outros monoterpenos em pequenas quantidades. A identificação de frações de álcoois, cetonas e substâncias oxigenadas em geral, utilizando dados de comparação com espectroteca e índices de retenção, é de baixa confiabilidade. Desta forma, foram citadas apenas as substâncias que apresentaram altos índices de similaridade entre espectros na comparação com a espectroteca.

A fração $\mathrm{F}_{3}$ contém ácidos graxos, misturas de esteróis e os diterpenos do tipo clerodano CTN, ciscajucarina B (c-CJC-B) e trans-cajucarina B (t-CJC-B) (Figura 1) previamente isolados das cascas do caule de Croton cajucara (Maciel et al., 1998a,b, 2003). O somatório de esteróis e diterpenos no cromatograma constituiu cerca de $40 \%$ do total de $\mathrm{F}_{3}$. A fração $\mathrm{F}_{4}$ contém majoritariamente os clerodanos isoméricos cis e trans-cajucarina B. Os clerodanos CTN, c-CJC-B e t-CJC-B foram identificados nas frações $\mathrm{F}_{3}$ e $\mathrm{F}_{4}$, via coinjeção com padrões autênticos (Figura 2) . A Figura 3 apresenta o espectro de massas da CTN e as Figuras 4 e 5 apresentam respectivamente, os espectros de massas dos isômeros c-CJC-B e t-CJC-B detectados no cromatograma da fração $\mathrm{F}_{3}$. Os Esquemas 2 e 3 mostram fragmentos característicos que foram atribuídos aos clerodanos transcrotonina (CTN) e cajucarinas B (t-CJC-B e c-CJC-B), respectivamente.

\section{Propriedade antifúngica do óleo fixo obtido das cascas do caule de Croton cajucara}

O controle positivo representa em experimentos in vitro uma condição básica para o crescimento de fungos. Neste tratamento o fungo é submetido ao meio de cultura BDA acrescido de antibiótico, para evitar o crescimento de bactérias. O controle negativo, também conhecido como testemunha padrão ou branco, é preparado da mesma forma, entretanto, com uma diferença: a adição de $1 \%\left(v^{\prime} v^{-1}\right)$ de DMSO. A utilização do DMSO foi necessária para solubilizar o óleo fixo de Croton cajucara no meio de cultura, constituído principalmente por água, permitindo, desta maneira, que o óleo seja difundido igualmente no meio de cultura. A utilização do controle negativo permite que os efeitos dos tratamentos sejam subtraídos de um possível efeito do DMSO, sobre o crescimento dos fungos. 
<smiles>CC(=O)C1C[C@@H]2C[C@@H]1C[C@H]2C(C)=O</smiles><smiles>CC1=CC(=O)CC2[C](CCc3ccoc3)C(C)CCC12</smiles><smiles>CC1=CC(=O)CC23CC2C(C)CCC13</smiles>

$330[\mathrm{M}]^{+}$ $\mathrm{m} / \mathrm{z} 271$<smiles>CC(C)C(C)C</smiles>

$\mathrm{m} / \mathrm{z} 189$

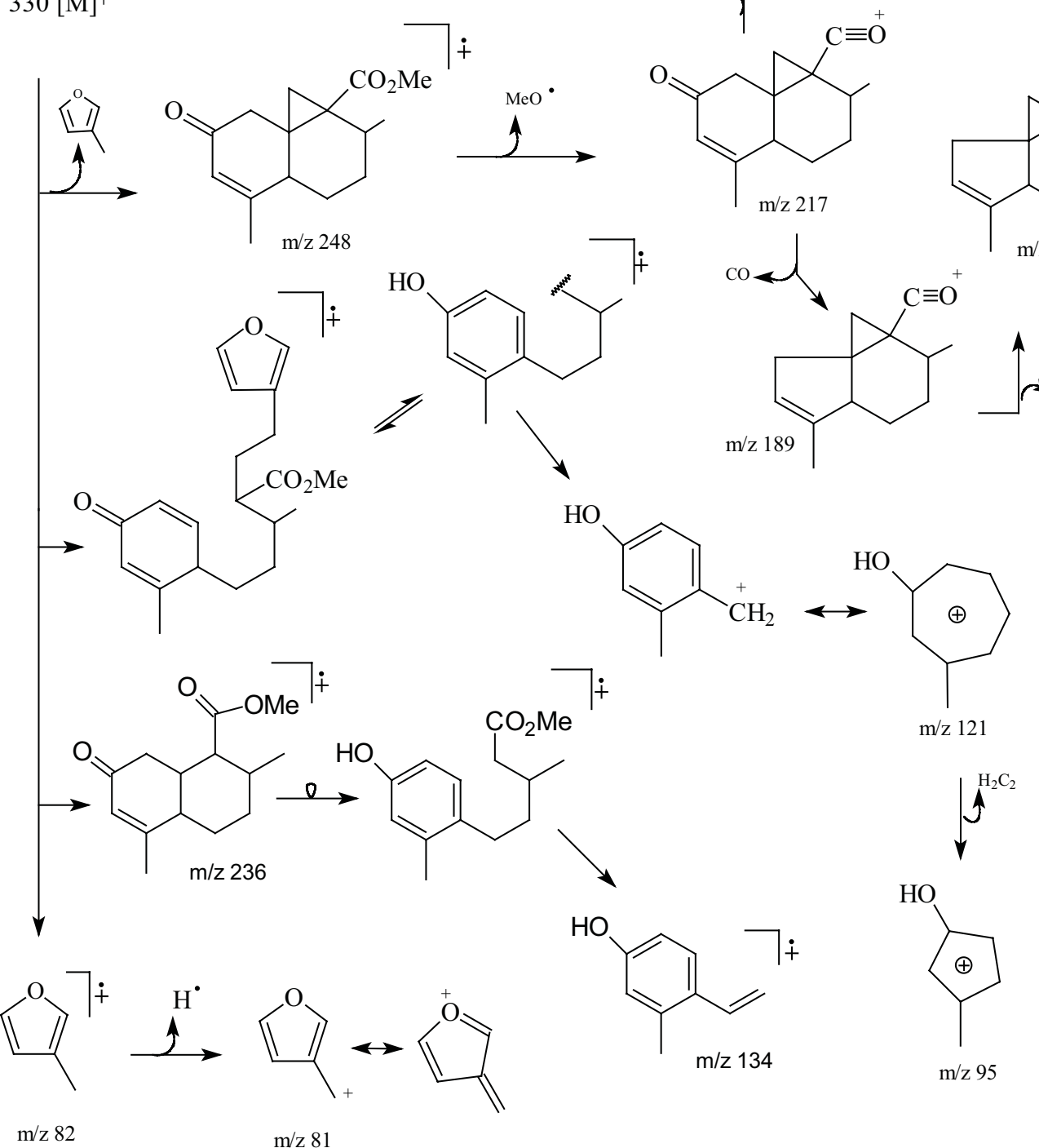

Esquema 3. Proposta de fragmentação da cis e trans-cajucarinas B.

A comparação feita entre o controle testemunha padrão (BDA + DMSO) e o controle positivo (BDA) nas placas de Petri, com o fungo Fusarium oxysporum, observou-se que não houve diferenças significativas durante os cinco dias de ensaio. No entanto, os fungos Rhizoctonia solani e Sclerotium rolfsii, apresentaram sensibilidade à presença do DMSO no meio de cultura. Por esta razão, os percentuais de inibição foram trabalhados a partir dos resultados obtidos com a testemunha padrão. Desta forma, o efeito do DMSO sobre o crescimento dos fungos foi subtraído. Uma vez subtraído os efeitos inibitórios do DMSO sobre os fungos Rhizoctonia solani e Sclerotium rolfsii, os tratamentos com o óleo fixo de Croton cajucara obtido via procedimento cromatográfico 


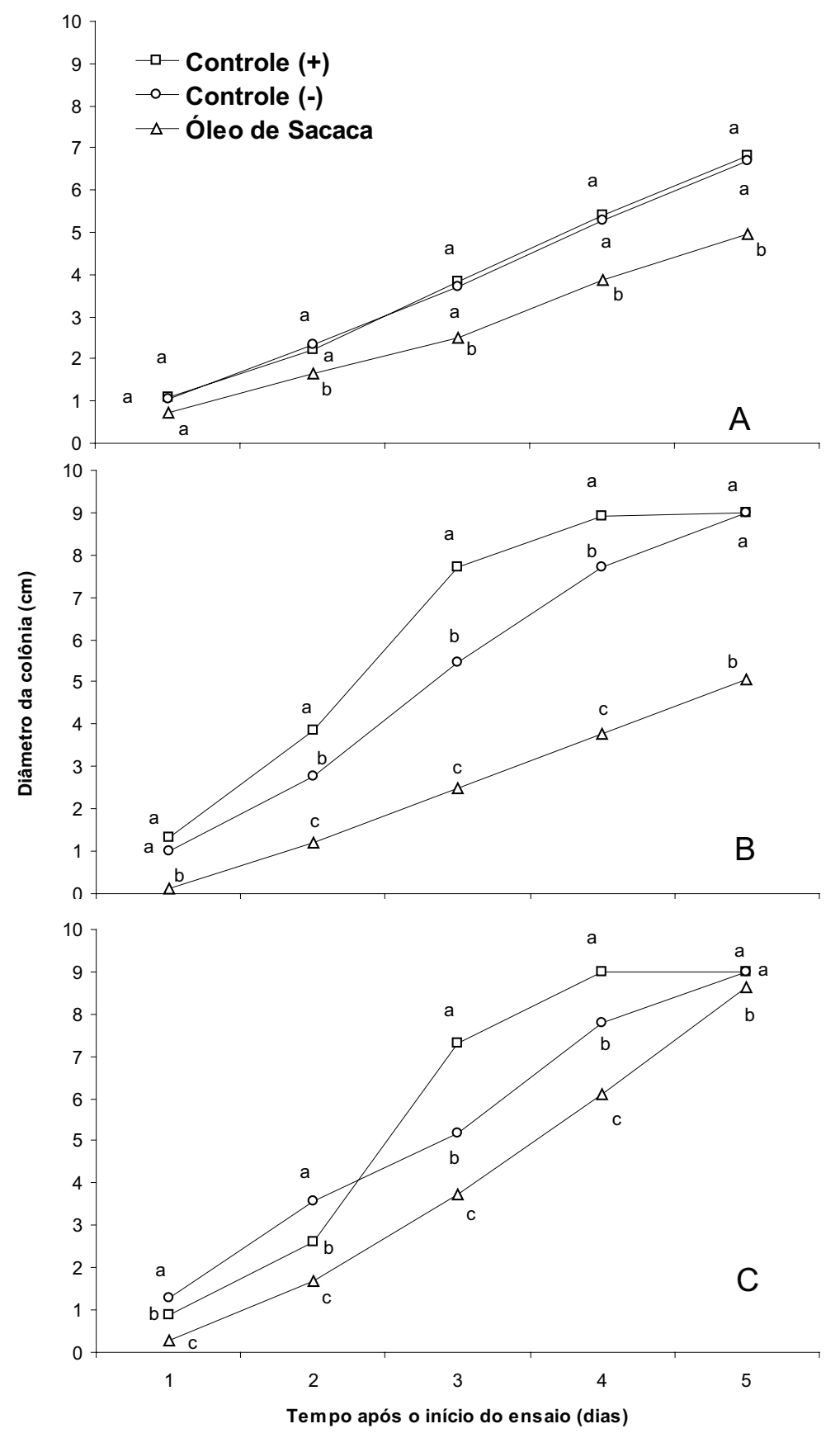

Figura 6. Diâmetro de crescimento das colônias em meio de cultura BDA, durante cinco dias. Controle positivo: meio de cultura BDA; Controle testemunha padrão: meio de cultura $\mathrm{BDA}+1 \%$ DMSO; óleo fixo de Croton cajucara: meio de cultura BDA com concentração 0,2 mg. $\mathrm{mL}^{-1}$ de óleo fixo e 1\% de DMSO; A) Fusarium oxysporum.; B) Rhizoctonia solani.; C) Sclerotium rolfsii.

(OF-CC proveniente da reunião de frações $\mathrm{F}_{\mathrm{AB}}$ ) apresentaram efeito inibitório suplementar ao observado na testemunha padrão (Figuras 6 e 7). Resultados semelhantes foram encontrados por Souza et al. (2003) ao verificarem a atividade biológica do óleo de capim limão sobre o desenvolvimento dos mesmos tipos de fungos utilizados neste trabalho.
Após cinco dias de observação, as placas de Petri contendo o fungo Sclerotium rolfsii, apresentaram uma possível alteração fisiológica, já que as estruturas de resistência, denominadas de esclerócios, apresentaramse rugosas, com manchas variando do marrom-escuro ao marrom-claro e diâmetro quatro vezes superior ao encontrado nas placas do controle positivo. Ao se avaliar 

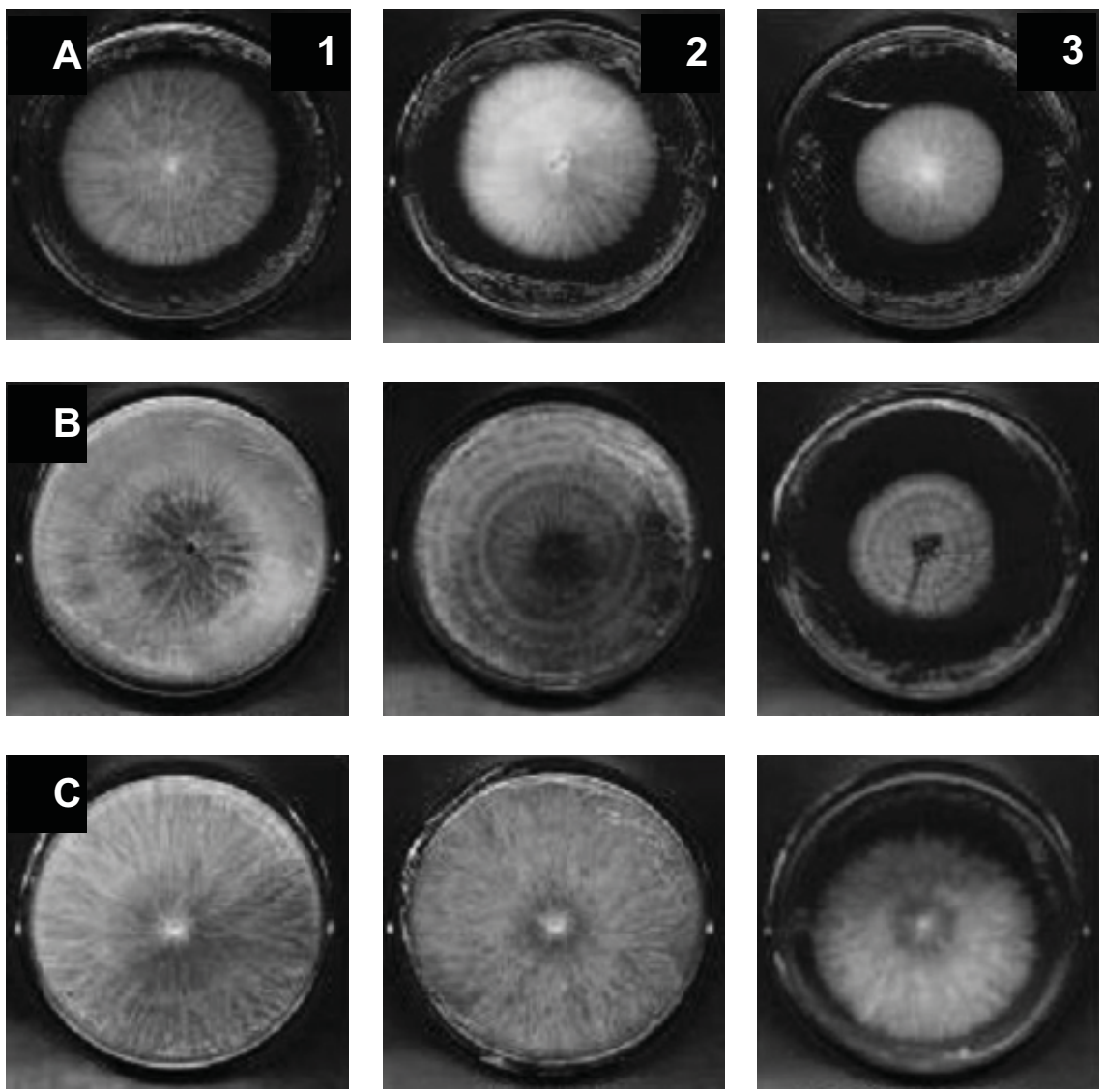

Figura 7. Colônias dos fungos crescidos em meio de cultura BDA. Imagens capturadas no quinto dia após o início do ensaio, onde nas colunas: 1) meio de cultura BDA; 2) meio de cultura BDA + 1\% DMSO e 3) meio de cultura BDA com concentração de $0,2 \mathrm{mg} \cdot \mathrm{mL}^{-1}$ do óleo fixo de Croton cajucara e $1 \%$ de DMSO; nas linhas: A) Fusarium oxysporum.; B) Rhizoctonia solani e C) Sclerotium rolfsii.



Figura 8. Percentual de inibição do crescimento das colônias de Fusarium oxysporum, Rhizoctonia solani e Sclerotium rolfsii em meio de cultura BDA, contendo 0,2 mg.mL${ }^{1}$ do óleo fixo de Croton cajucara, durante cinco dias. 
os efeitos do OF-CC sobre o crescimento do fungo, verificou-se efeito fungistático em decorrência de redução no crescimento da colônia, tendo sido comprovado inibição do crescimento dos três fungos testados (Figuras 6 e 7). Em contrapartida, a resposta fisiológica dos fungos testados (na presença do OF-CC no meio de cultura) foi diferente, tendo variado de acordo com o tipo de fungo testado. Observou-se que os fungos Rhizoctonia solani e Sclerotium rolfsii foram resistentes ao óleo testado, de modo que o percentual de inibição diminuiu com o transcorrer dos dias, principalmente para Sclerotium rolfsii. Como pode ser observado na Figura 6, o fungo Fusarium oxysporum foi o que sofreu efeito inibitório mais estável ao longo dos 5 dias de crescimento. Comparativamente, o fungo Sclerotium rolfsii sofreu a maior queda do efeito inibitório, enquanto o fungo Rhizoctonia solani sofreu maior inibição ao crescimento da colônia (Figura 8).

\section{CONCLUSÃO}

A presença de sesquiterpenos foi comprovada no óleo fixo obtido das cascas do caule de Croton cajucara Benth, tendo sido evidenciado que o $\alpha$-copaeno $(20,1 \%)$ e cipereno (21,8\%) são os componentes majoritários (70\% de percentual sesquiterpénico total) deste óleo. Dentre os sesquiterpenos minoritários mais oxigenados, destaca-se o linalol. Adicionalmente, foram confirmadas as presenças de ácidos graxos, misturas de esteróis e os diterpenos bioativos trans-crotonina, cis-cajucarina $\mathrm{B}$ e trans-cajucarina B.

Com relação aos ensaios in vitro pode-se dizer que o DMSO serviu apenas como veículo nos ensaios in vitro com fungos, como observado por Souza et al (2003), Ferreira et al. (2004) e Jacques et al. (2005), tendo sido necessário um estudo prévio das concentrações ideais para a sua utilização. O óleo fixo testado na concentração de $0,2 \mathrm{mg} \cdot \mathrm{mL}^{-1}$, apresentou atividade biológica de grande valor agronômico, já que possui sesquiterpenos e clerodanos que podem ser utilizados isoladamente em testes posteriores, bem como disponibilizados em mistura (óleo fixo) para uso comercial na agricultura.

Equívocos com relação ao uso tradicional de Croton cajucara, onde folhas são comercializadas com indicações terapêuticas de cascas, encontram-se documentados (Veiga Jr. et al., 2005). Desta forma, trabalhos que enfoquem a constituição química de óleos provenientes de folhas ou cascas deste Croton, bem como investigações biológicas, devem ser divulgados objetivando esclarecimentos que contribuam com o uso tradicional desta espécie medicinal, com seus estudos etnobotânico, etnofarmacológico, fitoquímico e fitoterápico. Adicionalmente, esta planta representa o Croton mais estudado no mundo, detendo cerca de 60 artigos publicados, sendo, portanto, uma das plantas medicinais nacionais com grande repercussão internacional. $\mathrm{O}$ processo de validação científica dos benefícios terapêuticos de Croton cajucara, só está sendo possível, porque estudos multidisciplinares estão sendo desenvolvidos (Maciel et al., 2002a,b, 2000, 2006).

\section{AGRADECIMENTOS} da CAPES.

Os autores agradecem ao Programa PRODOC

\section{REFERÊNCIAS}

Agner AR, Maciel MAM, Pinto AC, Cólus ISM 2001. Antigenotoxicity of trans-dehydrocrotonin, a clerodane diterpene from Croton cajucara. Planta Med 67: 815-819.

Almeida ABA, Miotto AM, Nunes DS, Spadari-Bratifisch RC, Souza-BritoARM2002. Mechanism of antiulcerogenic activity of semi-synthetic crotonin obtained from Croton cajucara Benth. Rev Bras Farmacogn 12(Supl. 1): $105-110$.

Almeida ABA, Melo PS, Hiruma-Lima CA, Gracioso JS, Carli L, Nunes DS, Haun M, Souza Brito ARM 2003. Antiulcerogenic effect and cytotoxic activity of semisynthetic crotonin obtained from Croton cajucara Benth. Eur J Pharmacol 472: 205-212.

Alviano WS, Mendonça-Filho RR, Alviano DS, Bizzo HR, Souto-Padrón T, Rodrigues ML, Bolognese AM, Alviano CS, Souza MMG 2005. Antimicrobial activity of Croton cajucara Benth linalool-rich essential oil on artificial biofilms and planktonic microorganisms. Oral Microbiol Immunol 20: 101-105.

Araújo VC, Corrêa GC, Maia JGS, Silva ML, Gottlieb OR, Marx MC, Magalhães MT 1971. Óleos essenciais da Amazônia contendo linalol. Acta Amazônica 1: 4547.

Bighetti EJ, Hiruma-Lima CA, Gracioso JS, Souza Brito ARM 1999. Antiinflammatory and antinociceptive effects in rodents of the essential oil of Croton cajucara Benth. J Pharm Pharmacol 51: 1447-1453.

Ferreira MA, Souza MAA, Araujo OJL, Ferreira ABB, Stark EMLM, Souza SR 2004. Efeito do lapachol sobre o desenvolvimento dos fungos Fusarium oxysporium, Sclerotium Rolfsii e Rhizoctonia solani. Anais da XIV Jornada de Iniciação Cientifica da Universidade Federal Rural do Rio de Janeiro. Seropédica, RJ, Brasil.

Hiruma-Lima CA, Gracioso JS, Nunes DS, Souza Brito ARM 1999a. Effects of an essential oil from the bark of Croton cajucara Benth. on experimental gastric ulcer models in rats and mice. J Pharm Pharmacol 51: 341346.

Hiruma-Lima CA, Spadari-Bratfisch RC, Grassi-Kassisse DM, Souza Brito ARM 1999b. Antiulcerogenic mechanisms of dehydrocrotonin, a diterpene lactone obtained from Croton cajucara. Planta Med 65: 325330.

Hiruma-Lima CA, Gracioso JS, Rodríguez JA, Haun M, Nunes DS, Souza Brito ARM 2000a. Gastroprotective effects of essential oil from the bark of Croton cajucara Benth (Euphorbiaceae). J Ethnopharmacol 69: 229-234.

Hiruma-Lima CA, Gracioso JS, Rodríguez JA, Bighetti EJB, Grassi-Kassisse DM, Nunes DS, Souza Brito ARM 2000b. Effect of essential oil obtained fom Croton 
cajucara Benth. on gastric ulcer healing and protective factors of the gastric mucosa. Phytomedicine 9: 523529.

Jacques M, Ramos RG, Ferreira MA, Araújo OJL, Souza MAA, Stark EMLM, Ferreira ABB, Souza SR 2005. Atividade biológica da lapachona extraído do Ipê roxo e seus análogos sintéticos sobre o crescimento de Fusarium oxysporum. Anais da XV Jornada de Iniciação Científica da Universidade Federal Rural do Rio de Janeiro. Seropédica, RJ, Brasil.

Lemos TLG, Machado MIL, De Menezes JESA, De Sousa CR 1999. Essential oil of Croton cajucara Benth. J Essent Oil Res 11: 411-412.

Lopes D, Bizzo HR, Sá Sobrinho AF, Pereira MVG 2000. Linalool-rich essential oil from leaves of Croton cajucara Benth. J Essent Oil Res 12: 705-708.

Maciel MAM, Pinto AC, Brabo SN, Silva MN 1998a. Terpenoids from Croton cajucara. Phytochemistry 49: 823-828.

Maciel MAM, Pinto AC, Brabo SN, Arruda AC 1998b. Estudo da variação dos teores de terpenóides bioativos isolados das cascas do caule de Croton cajucara, nativos e cultivados no estado do Pará. Rev Univ Rural, Sér Ciênc Exatas e da Terra 18/20: 17-34.

Maciel MAM, Pinto AC, Arruda AC, Pamplona SGSR, Vanderlinde FA, Lapa AJ, Cólus IMS, Echevarria A, Grynberg NF, Farias RAF, Luna-Costa AM, Rao VSN 2000. Ethnopharmacology, phytochemistry and pharmacology: a successful combination in the study of Croton cajucara. J Ethnopharmacol 70: 41-55.

Maciel MAM, Pinto AC, Veiga Jr. VF 2002a. Plantas medicinais: a necessidade de estudos multidisciplinares. Quim Nova 25: 429-438.

Maciel MAM, Pinto AC, Veiga Jr VF, Martins JR, Grynberg NF, Echevarria A, Lapa AJ, Vanderlinde FA 2002b. Croton cajucara as an alternative to traditional medicine in a modern health system, Phytochem. Pharmacol. II Ser. Recent Prog. Med. Plants 8: 459-475.

Maciel MAM, Pinto AC, Kaiser CR 2003. NMR and structure review of some natural furoclerodanes. Magn Reson Chem 41: 278-282.

Maciel MAM, Castro-Dantas TN, Câmara JKP, Pinto AC, Veiga Jr VF, Kaiser CR, Pereira NA, Carneiro CMTS, Vanderlinde FA, Lapa AJ, Agner AR, Cóllus IMS, Echevarria-Lima J, Grynberg NF, Esteves-Souza A, Pissinate K, Echevarria A 2006. Pharmacological and biochemical profiling of lead compounds from traditional remedies: the case of Croton cajucara. In: Advances in Phytomedicine (vol. 2) (Lead molecules from natural products, Discovery and New Trends; Eds. Khan, M. T. H. , Ather, A.), chapter 14, pp 229257.

Rosa MDS, Mendonca RR, Bizzo HR, Rodrigues ID, Soares RMA, Souto-Padron T, Alviano CS, Lopes AHCS 2003. Antileishmanial activity of a linalool-rich essential oil from Croton cajucara. Antimicrob Agents Ch. 47: 1895-1901.

Silva RM, Santos FA, Maciel MAM, Pinto AC, Rao VSN 2001a. Effect of trans-dehydrocrotonin, a 19-nor-clerodane diterpene from Croton cajucara on experimental hypertriglyceridaemia and hypercholesterolaemis induced by Triton WR 1339 (tyloxapol) in mice. Planta Med 67: 763-765.

Silva RM, Santos FA, Rao VSN, Maciel MAM, Pinto AC 2001b.
The lipid-lowering effect of trans-dehydrocrotonin from Croton cajucara Benth. In mice fed on high-fat diet. J Pharm Pharmacol 53: 535- 539.

Silva RM, Oliveira FA, Cunha KMA, Maia JL, Maciel MAM, Pinto AC, Nascimento NRF, Santos FA, Rao VSN 2005. Cardiovascular effects of trans-dehydrocrotonin, a diterpene from Croton cajucara. Vasc Pharmacol 43: $11-18$.

Souza MAA, Bratti A, Stark EMLM, Souza SR 2003. Atividade biológica de óleo essencial de capim-limão (Cymbopogon citratus (D.C.) Stapf.) avaliada através da inibição miscelial de fungos fitopatogênicos. Anais do XXXVI Congresso Brasileiro de Fitopatologia. Uberlândia, MG, Brasil.

Veiga Jr VF, Pinto AC, Maciel MAM 2005. Plantas medicinais: cura segura? Quim Nova 28: 519-528.

Zacchi P, Pietsch A, Voges S, Ambrogi A, Eggers R, Jaeger P 2006. Concepts of phase separation in supercritical processing. Chem Eng Process 45: 728-733. 